\title{
ASPECTOS GERAIS E NÚMERO DE ETAPAS DO SISTEMA DE MEDICAÇÃO DE QUATRO HOSPITAIS BRASILEIROS ${ }^{1}$
}

\author{
Silvia Helena de Bortoli Cassiani \\ Adriana Inocenti Miasso ${ }^{3}$ \\ Ana Elisa Bauer de Camargo Silva \\ Flávio Trevisan Fakin ${ }^{5}$ \\ Regina Célia de Oliveira ${ }^{6}$
}

Cassiani SHB, Miasso AI, Silva AEBC, Fakin FT, Oliveira RC. Aspectos gerais e número de etapas do sistema de medicação de quatro hospitais brasileiros. Rev Latino-am Enfermagem 2004 setembro-outubro; 12(5):781-9.

Essa investigação identificou e analisou o sistema de medicação de 04 hospitais universitários, localizados nas cidades de Recife, Ribeirão Preto, Goiânia e São Paulo, após a aprovação nos Comitês de Ética em Pesquisa e da autorização da direção dos hospitais. Os dados foram coletados através de entrevista estruturada com um dos profissionais responsáveis pelo sistema de medicação e observação não participante e direta, por uma semana, nos vários sub-sistemas. Os resultados indicaram pontos que necessitam de aperfeiçoamentos como utilização de abreviações, falta de padronização de horários de administração de medicamentos, falta de informações atualizadas e completas do paciente, farmácia não funcionando 24 horas em um hospital, falta de centro de informações de medicamentos e outros. Evidenciou-se no hospital A 66 etapas, no $B$ 58 etapas, no $C 70$ etapas e no D 80 etapas do sistema de medicação. Simplificar os processos, diminuindo o número de etapas, é a chave para a redução de erros de medicação.

DESCRITORES: erros de medicação; sistemas de medicação; administração hospitalar

\section{GENERAL ASPECTS AND NUMBER OF PHASES OF THE MEDICATION} SYSTEM IN FOUR BRAZILIAN HOSPITALS

This study identified and analyzed the medication systems in 04 university hospitals located in Recife, Ribeirão Preto, Goiânia and São Paulo, Brazil, after approval by the Research Ethics Committee and authorization by the hospital directors. Data were collected through a structured interview with one of the professionals in charge of the medication system and nonparticipant and direct observation during one week. The results indicated the points requiring improvement, such as the use of abbreviations, lack of standardization in medication administration times, lack of updated and complete information about the patient, the pharmacy's not working 24 hours a day in hospitals and others. 66 phases were shown in Hospital A, 58 in B, 70 in $C$, and 80 in $D$ concerning the medication system. Simplifying the processes by reducing the number of phases is the key to reducing medication errors.

DESCRIPTORS: medication errors; medication systems; hospital administration

\section{ASPECTOS GENERALES Y NÚMERO DE ETAPAS DEL SISTEMA DE MEDICACIÓN EN CUATRO HOSPITALES BRASILEÑOS}

Esa investigación identificó y analizó el sistema de medicación en 04 hospitales universitarios en las ciudades de Recife, Ribeirão Preto, Goiânia y São Paulo, Brasil. Los datos fueron recopilados en dos etapas. En la primera etapa se realizó una entrevista estructurada con uno de los profesionales responsables por el sistema de medicación. En la segunda, se hizo observación no participativa y directa durante una semana. Los resultados indicaron los puntos que necesitan ser perfeccionados, tales como uso de abreviaciones, la falta de un padrón de horarios, falta de información actualizada y completa del paciente, farmacia que no funciona las 24 horas en un hospital, y otros. Se observó en el Hospital A 66 etapas, en el B 58 etapas, en el C 70 etapas y en el D 80 etapas respecto al sistema de medicación. Simplificar el proceso, diminuyendo el número de etapas, es la clave para reducir los márgenes de errores en la medicación.

DESCRIPTORES: errores de medicación; sistemas de medicación; administración hospitalaria

\footnotetext{
${ }^{1}$ Estudo patrocinado pela Fundação de Amparo à Pesquisa do Estado de São Paulo-FAPESP; ${ }^{2}$ Professor Associado, e-mail: shbcassi@eerp.usp.br; ${ }^{3}$ Professor Assistente, e-mail: amiasso@zipmail.com.br; ${ }^{4}$ Mestranda, e-mail: anaelisa@terra.com.br. Escola de Enfermagem de Ribeirão Preto, da Universidade de São Paulo, Centro Colaborador da OMS para o desenvolvimento da pesquisa em enfermagem; ${ }^{5}$ Enfermeiro do Hospital São Paulo, e-mail: ffakih@denf.epm.br; ${ }^{6}$ Doutoranda da Escola de Enfermagem/Escola de Enfermagem de Ribeirão Preto, da Universidade de São Paulo, e-mail: reginacusp@bol.com.br
} 


\section{INTRODUÇÃO}

Estimativas norte-americanas indicam que em 1997, 7000 pacientes hospitalizados tiveram reações adversas fatais aos medicamentos. Atualmente, em cada 100 admissões nos hospitais, há 6,5 eventos adversos à medicação e 5,5 eventos potenciais, sendo $28 \%$ desses evitáveis. Pacientes que sofreram de eventos adversos às medicações tiveram aumento na estadia hospitalar de 2 dias e um custo aumentado na internação de mais de 2000 dólares ${ }^{(1)}$.

Segundo relatório do Institute of Medicine (EUA), das 33,6 milhões de internações realizadas no ano de 1997, em hospitais dos EUA, por volta de 7000 americanos morreram devido a problemas causados por erros na medicação. Os erros ocorreram em 2 a $14 \%$ dos pacientes hospitalizados, sendo que $62 \%$ dos erros estiveram relacionados à prescrição médica e transcrição da mesma ${ }^{(2-4)}$.

Várias investigações sobre os erros de medicação estão sendo conduzidas atualmente, em nível mundial, analisando os sistemas de medicação de vários hospitais e avaliando os erros de forma sistêmica, buscando, assim, tirar o enfoque das causas dirigidas exclusivamente para os profissionais ${ }^{(5-7)}$. Especialistas e investigadores que há muito vêm se dedicando ao estudo dessa temática são unânimes em rejeitar as abordagens que enfocam os indivíduos, puramente, como a causa de erros e notificam que mudanças no sistema são a estratégia mais efetiva para reduzir a probabilidade de erros ${ }^{(7-8)}$. Um sistema adequado, visando a segurança organizacional do paciente, torna difícil aos profissionais errarem e permite a detecção e correção dos erros antes que eles venham a ocorrer, através de uma cultura de segurança pró-ativa ${ }^{(9)}$.

Esse estudo evidenciou o sistema de medicação e as falhas que influenciam o trabalho da equipe de saúde e determinam a ocorrência de erros de medicação em 4 hospitais brasileiros. Destaca-se a relevância prática e social dessa abordagem na medida em que inexistem, no país, dados oficiais acerca dos erros de medicação. 0 Brasil constitui o quinto país em consumo de medicamentos, o primeiro na América Latina, e a Fundação
Oswaldo Cruz estima em 24 mil mortes anuais por intoxicação medicamentosa ${ }^{(10)}$.

É, portanto, imperativo, atual e pioneiro lidar com a problemática dos erros de medicação entre os hospitais nacionais a fim de propor medidas para sua prevenção e nada melhor do que evidenciar, através de pesquisas, os problemas que já vêm sendo observados na prática.

\section{OBJETIVO}

Identificar e analisar o sistema de medicação em 04 hospitais brasileiros localizados nas cidades de Recife, Ribeirão Preto, Goiânia e São Paulo com respeito a aspectos que podem favorecer a ocorrência de erros na medicação.

\section{MATERIAL E METÓDOS}

Trata-se de estudo multicêntrico, do tipo survey exploratório, cujo propósito foi o de coletar descrições detalhadas da variável sistema de medicação em diferentes hospitais. A investigação foi realizada em unidades de clínica médica e farmácia de 04 hospitais universitários, localizados nas cidades de Recife, Ribeirão Preto, Goiânia e São Paulo, descritos no texto como Hospitais A, B, C e $D$, sendo que a seqüência das letras não corresponde àquela das cidades apresentadas. Essas instituições foram escolhidas por possuírem vínculo com universidades públicas estaduais ou federais, por fazerem parte da Rede Sentinela da ANVISA e por serem campo de estágio para as instituições formadoras de profissionais de saúde do país.

Os dados foram coletados em 2 etapas. $\mathrm{Na}$ primeira, foi realizada entrevista estruturada com um dos profissionais responsáveis pelo sistema de medicação, em cada um dos hospitais, com intuito de obter uma visão geral do sistema vigente. Utilizou-se o roteiro adaptado do estudo de Hatfield ${ }^{(11)}$, que identificava aspectos estratégicos, para a garantia da segurança de pacientes no que concerne à terapêutica medicamentosa como: tipo 
de prescrição médica, uso de horários padronizados, uso de um único tipo de bomba de infusão, informações atualizadas sobre os pacientes, entre outros.

Na segunda etapa, foram realizadas observações não-participantes e diretas durante uma semana em cada uma das etapas do sistema de medicação: prescrição médica, dispensação, preparo e administração de medicamentos. Três auxiliares de pesquisa permaneceram em cada um dos hospitais durante três semanas, após participarem de sessões de treinamento no total de 20 horas. As observações foram realizadas durante três dias no plantão manhã, dois dias no plantão tarde e dois dias no plantão noite em cada uma das etapas. Todas essas atividades ocorreram de forma simultânea nos hospitais.

Essa investigação foi realizada após a aprovação dos Comitês de Ética em Pesquisa e autorização da direção dos hospitais. Todos os participantes foram orientados sobre o estudo e assinaram o termo de consentimento livre e esclarecido.

\section{RESULTADOS E DISCUSSÃO}

Visão geral do sistema de medicação nos quatro hospitais

No que concerne à prescrição médica, observou-se que apenas um hospital (A) tem implementado a prescrição médica eletrônica e essa é feita utilizando o nome genérico dos medicamentos. No entanto, a prescrição manual ainda é bastante utilizada, nesse mesmo hospital, caso haja mudanças na condição dos pacientes e, conseqüentemente, alteração na prescrição prévia. Nos hospitais restantes, as prescrições médicas são manuais. No hospital B a prescrição é transcrita para o computador pelo enfermeiro ou escriturário, a fim de se requisitar o medicamento à farmácia. No hospital $C$ e $D$ utiliza-se a cópia carbonada da prescrição e nos hospitais B, C e D os medicamentos são prescritos utilizando tanto o nome genérico como o nome comercial. Todos os hospitais permitem a utilização de abreviações nas prescrições.

Observa-se, portanto, a predominância da prescrição manual e o uso tanto do nome comercial quanto do genérico nesses hospitais. Mesmo havendo a prescrição eletrônica, em um hospital, a enfermagem intervém transcrevendo ou enviando essa prescrição à farmácia. Destacam-se, nessas circunstâncias, aspectos importantes que podem ocasionar erros quais sejam: o fato das prescrições manuais dependerem da caligrafia do médico e interpretação da enfermagem, utilizações mistas de nomes comerciais e genéricos dos medicamentos na prescrição médica e uso de abreviações $^{(12)}$.

\section{Quanto ao horário padronizado na}

administração de medicamentos, em três dos hospitais analisados (hospital $\mathrm{A}, \mathrm{B}$ e $\mathrm{C}$ ) não há diretriz específica, dependendo da farmácia e horários de dispensação do medicamento, o hospital $D$ possui padronização nos horários. O ideal seria a padronização de horários em todo o hospital, inclusive para facilitar o serviço de farmácia e o da enfermagem no planejamento de suas atividades.

Em três hospitais ( $A, B$ e $C$ ) é permitida a utilização de mais de um tipo de bomba de infusão. Se for necessário comprar mais bombas a decisão pelo tipo será por questão econômica e não com o intuito de padronização. Sabe-se das dificuldades envolvidas com o uso de bombas de infusão de tipos diferentes, o que leva os profissionais da enfermagem a terem diferentes condutas nas infusões, dependendo do tipo de bomba utilizada $^{(13)}$.

Informações sobre o paciente como idade, peso, altura, alergia e diagnóstico do paciente estão disponíveis nos prontuários, porém, em diferentes lugares e, em nenhum dos hospitais essas informações se encontram completas e atualizadas. Dados como, por exemplo, a alergia a determinados medicamentos deveriam estar em um local acessível e de destaque (como, por exemplo, em um sistema informatizado) e não no interior de um prontuário em local nem sempre acessível e desconhecido dos profissionais em geral ${ }^{(13)}$.

Quanto às informações sobre medicamentos para atualização dos profissionais, observa-se que há, nos hospitais, um formulário com nome dos medicamentos padronizados. Em alguns (A, B) os medicamentos estão distribuídos na listagem de acordo com o sistema corporal 
em que agem. No hospital B, as informações sobre os medicamentos são geralmente obtidas junto aos médicos e farmacêuticos, nas bulas dos medicamentos e no dicionário de especialidades farmacêuticas. Eventualmente tem-se acesso ao MICROMEDEX® (base de dados eletrônica sobre medicamentos). Nenhum desses hospitais possui centro de informações sobre medicamentos e também não há políticas para revisão das prescrições médicas para avaliação de terapêutica, alergias às medicações, interações medicamentosas e doses excessivas. Recentemente o hospital B iniciou o treinamento de farmacêuticos clínicos para que, futuramente, assumam essas funções. Em todos os hospitais analisados há comissão de padronização de medicamentos. Nota-se que não há nada formalizado que auxilie os profissionais nas dúvidas sobre medicamentos, ao contrário do que a literatura indica ${ }^{(13-}$ 14)

Quanto ao horário de funcionamento da farmácia, somente em um dos hospitais (D) a farmácia não funciona durante 24 horas do dia. Em todos os hospitais, farmacêuticos fornecem informações para os profissionais a respeito de medicamentos novos ou não padronizados, se solicitados, e durante os momentos em que estão no setor.

Sabe-se que a farmácia hospitalar precisa estar aberta nas 24 horas do dia e contar com farmacêuticos. O ideal é ainda ter a presença de farmacêuticos clínicos participando das visitas médicas e revendo as prescrições $^{(14)}$.

Em três dos hospitais (A, C e D) as doses são enviadas às clínicas na forma individualizada e todos os hospitais possuem mini-estoque de medicamentos nas áreas críticas e não críticas. Destaca-se que nenhum dos hospitais conta com o sistema de distribuição de medicamentos em dose unitária, embora já seja positivo o fato de eliminar as farmácias satélites, tão utilizadas no passado. A meta deve ser a mudança do sistema de distribuição para o de dose unitária, que é considerada uma medida para otimizar o trabalho da enfermagem e reduzir os erros de medicação ${ }^{(6-7)}$. Não é interessante haver mini-estoque face à inconveniência de estocar medicamentos caros, devido aos prazos de validade e acúmulo de estoques em algumas áreas com prejuízo de outras.

Nesses hospitais, pelo menos $80 \%$ da nutrição parenteral e quimioterapia, infusões intermitentes e contínuas são preparadas pela farmácia ou outro serviço.

Todos os hospitais informaram que não há dupla conferência na dispensação de medicamentos por diferentes profissionais da farmácia.

Embora haja diretriz para que em todos os hospitais os medicamentos prescritos e suspensos devam retornar à farmácia, não há controles e auditorias regulares para conferir se as devoluções estão realmente ocorrendo. Somente um dos hospitais (A) usa códigos de barras na documentação e dispensação de medicamento. Nenhum dos hospitais utiliza diretrizes escritas para o uso de medicações por pacientes em casa e para auto-administração de medicações por pacientes. Denota-se, assim, algumas falhas no sistema que podem facilitar o erro de medicação e além dos já mencionados a falta de horários padronizados da administração de medicamentos, a falta de informações importantes do paciente atualizadas e disponíveis como, por exemplo, alergias; falta de informação nas clínicas sobre medicamentos, utilização de mais de um tipo de bomba de infusão, entre outros.

Além disso, em todos os hospitais envolvidos na pesquisa não existe um comitê formal que investiga erros na medicação, dois desses ( $A$ e B) possuem um método para relato desses erros e, em todos os hospitais, nem a farmácia, nem a enfermagem e nem a equipe médica recebe relatos periódicos de erros em medicação. Esse é um alerta de que mudanças são necessárias, já que o sistema de notificações de erros deve ser visto como uma função das equipes de Controle de Qualidade ou de outra Comissão especial.

A Tabela 1 sintetiza os resultados encontrados em cada um dos hospitais. 
Tabela 1 -Análise sintética das etapas do sistema de medicação analisadas em quatro hospitais. Ribeirão Preto, 2002

\begin{tabular}{|c|c|c|c|c|}
\hline Itens & $\begin{array}{l}\text { Hospital } \\
\text { A }\end{array}$ & $\begin{array}{l}\text { Hospital } \\
\text { B }\end{array}$ & $\begin{array}{l}\text { Hospital } \\
\text { C }\end{array}$ & $\begin{array}{c}\text { Hospital } \\
\text { D }\end{array}$ \\
\hline 1 - Prescrição eletrônica em hospitais & $\mathrm{X}$ & & & \\
\hline $\begin{array}{l}1 \text { a - Medicamentos prescritos pelo nome genérico } \\
1 \text { b - Abreviaçóes permitidas }\end{array}$ & $\begin{array}{l}\mathrm{x} \\
\mathrm{x}\end{array}$ & $\begin{array}{l}\mathrm{x} \\
\mathrm{x}\end{array}$ & $\begin{array}{l}\mathrm{x} \\
\mathrm{x}\end{array}$ & $\begin{array}{l}\mathrm{x} \\
\mathrm{x}\end{array}$ \\
\hline 1c - Horários padronizados pelo sistema para administraçâo de medicamentos de rotina & & & & $\mathrm{x}$ \\
\hline $\begin{array}{l}\text { 1d - Idade do paciente, peso, altura, alergias e diagnóstico disponiveis no sistema/prontuário } \\
\text { de forma padronizada pelo sistema }\end{array}$ & & & & $\mathrm{x}$ \\
\hline $\begin{array}{l}\text { 1e - Informaçóes completas e atualizadas } \\
2 a-\text { Farmácia aberta } 24 \text { horas por dia }\end{array}$ & $\mathrm{X}$ & $\mathrm{X}$ & $\mathrm{X}$ & \\
\hline $2 \mathrm{~b} 1$ - Pelo menos $90 \%$ das doses enviadas na forma de dose individualizada & $\mathrm{x}$ & & $\mathrm{x}$ & $\mathrm{x}$ \\
\hline \multicolumn{5}{|l|}{2 b2 - Pelo menos $90 \%$ das doses enviadas na forma de dose unitária } \\
\hline $2 c$ - Mini-estoque nas áreas de cuidado crítico e não crítico & $\mathrm{x}$ & $\mathrm{x}$ & $\mathrm{x}$ & $\mathrm{x}$ \\
\hline $\begin{array}{l}\text { 2d - Farmacêuticos fornecem informaçâo para os profissionais a respeito de medicamentos } \\
\text { novos ou não padronizados no hospital }\end{array}$ & & $\mathrm{X}$ & & \\
\hline $\begin{array}{l}2 \ominus \text { - Pelo menos } 80 \% \text { da nutriçâo parenteral e quimioterapia, infusóes intermitentes } \theta \\
\text { contínuas são preparados pela farmácia ou outro serviço }\end{array}$ & $\mathrm{x}$ & $\mathrm{x}$ & $\mathrm{x}$ & $\mathrm{x}$ \\
\hline \multicolumn{5}{|l|}{$2 f$ - Centro de informaçбes sobre medicamentos } \\
\hline \multicolumn{5}{|l|}{ 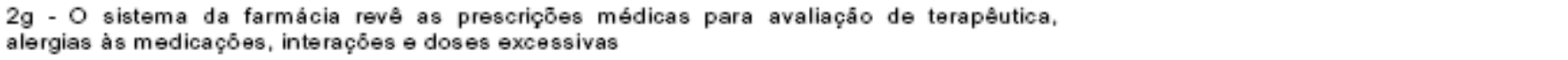 } \\
\hline \multicolumn{5}{|l|}{$\begin{array}{l}2 \mathrm{~h} \text { - Dupla conferância por profissionais da farmácia na dispensaçâo dos medicamentos por } \\
\text { diferentes profissionais }\end{array}$} \\
\hline $2 \mathrm{i}$ - Comissão de padronização de medicamentos & $\mathrm{x}$ & $\mathrm{x}$ & $\mathrm{x}$ & $\mathrm{x}$ \\
\hline \multicolumn{5}{|l|}{$\begin{array}{l}\text { 2j - Inform açס̄es atualizadas sobre os medicamentos disponiveis, fornecidos pela instituição a } \\
\text { pelos menos } 90 \% \text { das clínicas }\end{array}$} \\
\hline 3a - Procedimentos para evitar medicamentos estocados & $\mathrm{x}$ & $\mathrm{x}$ & & \\
\hline 3b-Códigos de barras usados na documentação da dispensaçâo de medicamentos & $\mathrm{x}$ & & & \\
\hline 3c- Permitido o uso de mais de uma marca de bomba de infusão no hospital & $\mathrm{X}$ & $\mathrm{x}$ & $\mathrm{x}$ & \\
\hline $\begin{array}{l}\text { 3d - Estoque de medicamentos (form alizado) nas clínicas } \\
4 \text { a - Comite que investiga erros na medicaçâo }\end{array}$ & $\mathrm{x}$ & $\mathrm{x}$ & & \\
\hline 4b - Método disponivel para relato de erros na medicação & $\mathrm{x}$ & $\mathrm{x}$ & & \\
\hline $\begin{array}{l}\text { 4c - Farmácia, enfermagem e a equipe médica recebem relatos períbdicos de erros na } \\
\text { medicaçâo } \\
\text { 4d1 - Diretrizes escritas para: } O \text { uso de medicaçб̄es por pacientes em casa } \\
\text { 4d2 - A auto administraçâo de medicaçōes por pacientes }\end{array}$ & & & & \\
\hline $\begin{array}{l}\text { 4d3 - Dupla conferencia do cálculo de medicaçôes de alto risco } \\
\text { 4d4 - esclarecimento das prescriçoses para pacientes de alta hospitalar } \\
\text { 4d5 - Administração de medicamentos endovenosos }\end{array}$ & $\mathrm{x}$ & & & \\
\hline 4e - Devolução de medicamentos nâo utilizados & $\mathrm{x}$ & $\mathrm{x}$ & $\mathrm{x}$ & $\mathrm{x}$ \\
\hline $4 f$ - Formas especificas de controle de psicotrópico & $\mathrm{x}$ & $\mathrm{x}$ & & \\
\hline
\end{tabular}

$\mathrm{X}$ - presença

Fluxograma e descrição do processo de medicação por hospital

Na forma gráfica, consta, através de fluxogramas, o sistema de medicações dos quatro hospitais investigados, de forma sintética, já que o espaço da publicação não comportaria fluxos detalhados. Destacase que esses, com todos os processos do sistema de medicação, contam com 66 etapas no hospital A, 58 etapas no hospital B, 70 etapas no hospital C e 80 no hospital D.

Uma análise rápida revela a enorme quantidade de etapas e a responsabilidade dos vários profissionais envolvidos. Por se tratar de um sistema multidisciplinar, uma falha em algum momento pode desencadear atos falhos em todo o processo subseqüente. Assim, falhas na prescrição podem atrasar a dispensação e, conseqüentemente, a administração dos medicamentos aos pacientes, além de requisitar trabalho extra do pessoal envolvido. Simplificar o processo nesses hospitais é, portanto, a chave para a redução dos erros ${ }^{(4)}$.

Várias são as estratégias que podem simplificar o processo. Uma dessas seria reduzir o número de papéis requeridos para um processo ser completado. Por exemplo, se a prescrição médica eletrônica do hospital $A$ fosse enviada diretamente à farmácia, sem necessidade de revisão por um membro da equipe de enfermagem, transcrevendo-a ou anotando os horários, na mesma, poderia conferir mais rapidez ao processo, além de diminuir as chances de o erro ocorrer.

A instituição do sistema de distribuição de medicamentos por dose unitária e a padronização de horários da administração de medicamentos, por exemplo, seriam outros processos que trariam benefícios a todos, simplificariam o trabalho e trariam segurança ao paciente.

É, ainda, extremamente necessário contar mais efetivamente com a presença do farmacêutico nas clínicas, revendo prescrições, sanando dúvidas dos profissionais da equipe médica, enfermagem e pacientes e atuando 
em farmácias hospitalares abertas 24 horas por dia para promover a segurança de pacientes e economia para as instituições.

Os fluxogramas, apresentados a seguir, indicam os processos da prescrição médica, dispensação e administração de medicamentos dentro do sistema de medicação dos quatro hospitais. Esses fluxogramas, até então inexistentes nas unidades, possibilitarão a visão de como o sistema realmente funciona e o grande número de etapas existentes.

\section{Fluxograma do Sistema de Medicação do Hospital A}
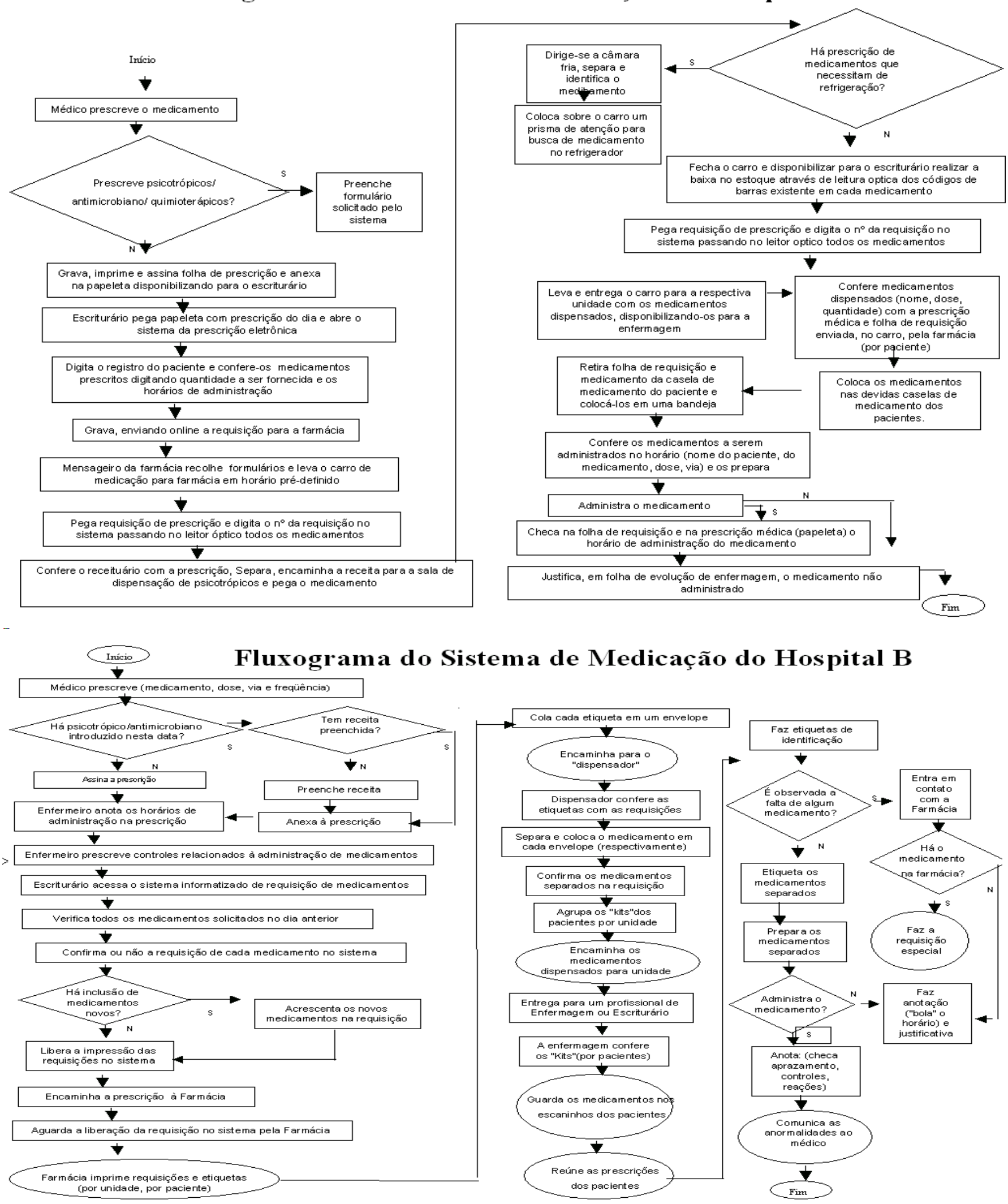

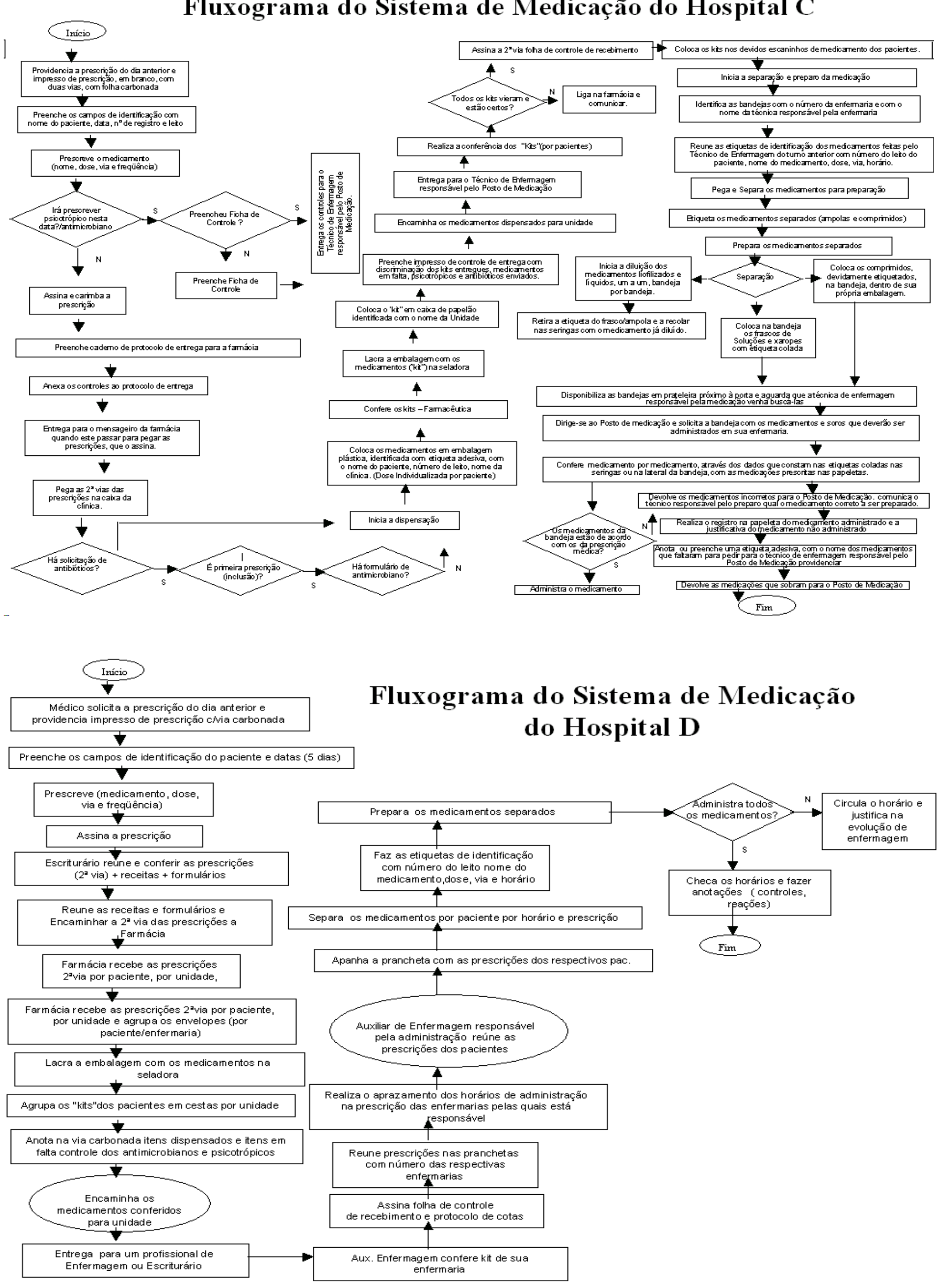


\section{CONCLUSÕES}

Há medidas de segurança de pacientes, no que concerne à terapêutica medicamentosa, já amplamente discutidas na literatura e ainda não observadas nos hospitais investigados. Uma dessas refere-se às informações sobre alergia medicamentosa que, embora coletadas, não estão visíveis para todos, não estando em um local específico para a anotação, podendo haver medicamentos prescritos para os quais o paciente já referiu alergia. Nem mesmo a farmácia tem informação desse aspecto. O hospital A teve a iniciativa de colocar um bracelete colorido no paciente como uma saída para alertar os profissionais, o que foi uma medida de segurança.

A presença do farmacêutico, auxiliando a equipe nas unidades, é uma iniciativa já demonstrada na redução de erros. Os fluxogramas apresentados mostram que o trabalho das farmácias está voltado basicamente à dispensação de medicamentos e ao preparo de nutrição parenteral e quimioterápicos. A revisão das prescrições pelos farmacêuticos poderia ser crucial na prevenção de erros de medicação. O ideal é que cada prescrição seja revista por um farmacêutico para assegurar a seleção apropriada de medicamentos, doses, vias e freqüência. Considerando que o número de farmacêuticos é pequeno nesses hospitais, pode-se pensar na revisão de uma amostra de prescrições de determinada unidade, por exemplo.

O papel dos enfermeiros também fica obscuro ao se analisar esses fluxogramas. Sabe-se que tais profissionais supervisionam o seu pessoal no processo da administração de medicamentos, mas falta atuação mais definida dentro do sistema.

Dentre os dados apresentados pelos hospitais não foram encontrados protocolos para as medicações. A literatura indica que pelo menos $75 \%$ das medicações devem ter protocolos e que os médicos devem estar envolvidos no seu desenvolvimento e aprovação, assim como as equipes de farmácia e de enfermagem devem ser estimuladas a manuseá-los, eliminando dúvidas que possam existir, já que essas equipes estão preparando e administrando os medicamentos ${ }^{(15)}$.

Acrescenta-se que há falta de informações disponíveis e atualizadas nas clínicas como livros, bulas, informes técnicos ou programas de computador. Interações medicamentosas e alimentares podem também estar ocorrendo devido à falta de informação escrita, protocolos e farmacêuticos, disponíveis, auxiliando as equipes médicas e de enfermagem.

O estoque formado nas clínicas por medicações não utilizadas e não devolvidas à farmácia pode colaborar para a ministração errônea a pacientes. Faltam políticas institucionais para o envio desses medicamentos e cobrança das equipes para a realização dessa atividade.

O sistema de distribuição de medicamentos por dose unitária, além de evitar erros é uma fonte de economia para a instituição. Embora já largamente difundida na literatura, nenhum desses grandes hospitais investigados utiliza esse sistema. A preparação e dispensação de medicamentos em locais não apropriados como postos de enfermagem, com falhas no conhecimento e na técnica dos profissionais, são práticas que podem estar levando a erros, na maioria das vezes não notificados.

O paciente tem um papel que deve ser incentivado, qual seja o de manter atualizado nas medicações que recebe e questionar e alertar a equipe quando os medicamentos estão indevidos. Políticas e diretrizes que incentivem a orientação dos pacientes tanto durante a internação como na alta hospitalar podem auxiliar na diminuição dos erros.

Este estudo determinou aspectos referentes à situação dos hospitais investigados no que concerne ao sistema de medicação. Identificou e analisou as falhas, a complexidade do sistema, a falta de atualização de processos já padronizados na literatura internacional, a deficiência de informações, enfim, a falta de segurança a que pacientes hospitalizados estão expostos no que concerne à terapêutica medicamentosa.

Importa, agora, que um comitê multidisciplinar, composto por farmacêuticos, enfermeiros e médicos, avalie cada etapa do sistema e traga idéias novas e criativas de como modificá-lo e simplificá-lo. Formar-se-á, assim, uma força tarefa com o objetivo de analisar o processo. Aplicar esse estudo em outros hospitais é uma sugestão para ampliar o debate nacional sobre a situação dos sistemas de medicação nos hospitais brasileiros. 


\section{REFERÊNCIAS BIBLIOGRÁFICAS}

1. Bates DW. Improving medication safety across institutions. J Qual Improv 2000; 26(6):319-20.

2. Gandhi T, Seger D, Bates DW. Identifyng drug safety issues: from research to practice. Int J Qual in Health Care 2000; 12(1):69-76.

3. Carvalho VT, Cassiani SHB. Erros na medicação e conseqüências para profissionais de enfermagem e clientes: um estudo exploratório. Rev Latino-Am Enfermagem jul/ago 2002; 10(4):523-9.

4. Kohn LT, Corrignan JM, Donaldson MS. To err is human. Building a safer health system. Washington (DC): National Academy Press; 2000.

5. Carvalho VT. Erros na administração de medicamentos: análise de relatos dos profissionais de enfermagem. [dissertação]. Ribeirão Preto (SP): Escola de Enfermagem de Ribeirão Preto/USP; 2000.

6. Cassiani SHB. Erros na administração de medicamentos: estratégias de prevenção. Rev Bras Enfermagem 2000; 53(3):424-30.

7. Anderson DJ, Webster CA. Systems approach to reduction of medication error on the hospital ward. J Adv Nurs 2001; 35(1):34-41.

8. Bates DW, Cohen M, Leape LL, Overhage JM, Shabot MM, Sheridan T. Reducing the frequency of errors in medicine using information technology. J Am Med Inform Assoc 2001; 8(4):299308.

9. Lee P. Ideal principles and characteristics a fail-safe medication use system. Am J Health Syst Pharm 2002; 59(4):369-71.

10. Morais J. A medicina doente. Isto é $2001 ; 15(5): 48-58$.

11. Hatfield, G. Rating hospitals for medication safety: a scorecard. Pharm Times 1999; 65(9):42-5.

12. Barker KN, Flyin EA, Pepper GA, Bates DW, Mikeal RL. Medication errors observed in 36 health care facilities. Arch Intern Med 2002; 162(16):1897-903.

13. Cohen MR. Medication errors: causes, prevention, and risk management. Washington (DC): Jones and Bartlett Publishers; 2000.

14. Leape LL, Cullen DJ, Clapp MD, Burdick E, Demonaco HJ, Erickson JI, Bates DW. Pharmacist participation on physician rounds and adverse drug events in the intensive care unit. JAMA 1999; 282(3):267-70.

15. Pepper GA. Errors in drug administration by nurses. Am J Health Syst Pharm 1999; 52(4):309-14. 